\title{
REFLEXIONES SOBRE LOS CONCEPTOS DE VALIDEZ Y EXISTENCIA DE LAS NORMAS JURÍDICAS*
}

\author{
Victoria Iturralde ** \\ Universidad del País Vasco
}

RESUMEN. El objeto de estas páginas es analizar algunas de las cuestiones que plantean los conceptos de validez y existencia de las normas en el ordenamiento. Respecto de la validez, planteo que los múltiples significados que dicho término tiene en la teoría jurídica pueden ser eliminados en favor del concepto de validez como legalidad. En este sentido un enunciado acerca de la validez es un enunciado interpretativo, y en muchos casos de carácter valorativo. A diferencia de la validez (que es una característica que las normas pueden o no tener) la noción de existencia plantea una la cuestión diferente: determinar en qué condiciones puede decirse que estamos ante una norma jurídica o, lo que es lo mismo, cuándo una norma jurídica existe. Para esto examino dos nociones de existencia: la de existencia formal y la de existencia convencional. cional.

Palabras clave: validez, legalidad, existencia, existencia formal, existencia conven-

ABSTRACT. The he aim of this work is to analyze some of the issues raised by the concepts of validity and existence of the rules in the legal order. About validity, I maintain that the multiple meanings that such a notion has in legal theory could be eliminated in favour of the concept of validity as legality. In this sense, a statement on validity is an interpretative one, and, in many cases, it has a value nature. Unlike the validity (which is a feature that the rules may or may not have), the existence poses a different question: to determine under what conditions it can be said that we have a legal rule or, to put it another way, when a legal rule exists. For this purpose, I analyze two concepts on existence: formal existence and conventional existence.

Keywords: validity, legality, existence, formal existence, conventional existence.

\footnotetext{
* Fecha de recepción: 7 de enero de 2008. Fecha de aceptación: 31 de enero de 2008.

** Ponencia presentada al XIII Congreso hispano-italiano de Teoría Jurídica, celebrado en Oñate (Guipúzcoa) los días 21 y 22 de septiembre de 2007. Agradezco sinceramente a todos los compañeros las sugerencias que me fueron realizadas y que, espero, hayan contribuido a mejorar aquellas reflexiones.
} 


\section{PLANTEAMIENTO}

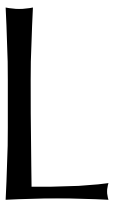

a cuestión de la validez es un tema omnipresente en la teoría del Derecho tanto desde el punto de vista de la validez externa (validez del ordenamiento), como desde la perspectiva de la validez interna) (validez en el ordenamiento). En estas páginas me referiré únicamente a esta segunda cuestión, respecto de la cual expondré la pertinencia de limitar el concepto de validez a la legalidad del acto de producción normativa y, que un juicio de validez es un juicio interpretativo que, en muchos, casos tiene carácter valorativo.

A diferencia de lo que ocurre con la validez, el tema de la existencia de las normas, no ha sido objeto de análisis tan precisos por parte de la teoría del Derecho, no obstante su doble importancia. De un lado, desde el punto de vista de la estructura de los ordenamientos jurídicos, éstos están formados por normas existentes. De otro, son las normas existentes las prima facie aplicables, «la inexistencia se traduce en la inaplicación de la norma por parte de los operadores jurídicos: una norma inexistente no necesita someterse a un juicio específico de invalidez» ${ }^{1}$. Las normas existentes gozan de una presunción de validez, presunción que puede cuestionarse sometiendo al órgano judicial correspondiente la «cuestión de validez», o bien inaplicar la norma en cuestión.

\section{VALIDEZ}

\subsection{Validez: un término ambiguo}

Comenzaré dando breve cuenta de los múltiples significados que tiene este término en la teoría del Derecho.

Según WRÓBLEWSKI validez es un término que denota tres conceptos distintos, conceptos que (en una terminología que se ha convertido en lugar común) denomina validez sistémica, validez factual y validez axiológica ${ }^{2}$.

a) Validez sistémica ${ }^{3}$ una norma $(n)$ es válida en un sistema jurídico $(s j)$ si se cumplen las condiciones siguientes: a) $n$ ha sido promulgada de acuerdo con las normas válidas en $s j$ y por tanto ha entrado en vigor, o $b$ ) $n$ es una consecuencia reconocida de las normas válidas en $s j ; c) n$ no ha sido formalmente rechazada («derogada»); $d$ ) $n$ no es inconsistente con las normas válidas en $s j ; e) n$ no es inconsistente con ninguna norma válida en sj entonces: $\left.e_{1}\right) n$ no es considerada inválida de acuerdo con las normas sobre

1 M. GASCÓN, 1997: «Sentido y alcance de algunas distinciones sobre la invalidez de las leyes», p. 148.

2 J. WRÓBLEWSKI, 1992: The Judicial Application of Law, pp.77-85 y J. WRÓBLEWSKI, 1982: «Tre concetti di validità», pp.585-595. A estos tres conceptos de validez se refieren también A.G. CONTE, 1975: «Validità», p. 421 (validez formal, validez ideal o axiológica y validez material); F. OsT, 1988: «Validité», quien habla de validez formal (asociada el término legalidad), validez empírica (asociada a efectividad) y validez axiológica (asociada a legitimidad); A. AARNIO, 1991: Lo racional como razonable, pp. 718-787.

${ }^{3}$ El concepto de validez sistémica, corresponde con los de «legal validity» (G.H. vON WRIGHT), «validez intra-sistémica» (U. SCARPELLI), «validez constitucional» (R. SCHREIBER) y «concepción formal de la validez» (L. NOWAK). 
conflicto de normas, $\left.\mathrm{o} e_{2}\right) n$ es interpretada de tal manera que sea eliminada la inconsistencia en cuestión.

b) Validez factual. Hay dos versiones del concepto de validez factual. Según la versión moderada, la validez factual consiste en la validez sistémica a la que hay que añadir una condición ulterior: que la norma no haya sido derogada por desuetudo. Esto significa que las normas validas (validez sistémica) que no han sido aplicadas por los órganos competentes del Estado durante un periodo de tiempo suficientemente amplio pierden su validez. Según esta idea, son normas válidas sólo las que son usadas en decisiones operativas. Según la versión radical, una norma válida es una norma que está siendo utilizada, es decir, que se refiere o que se aplica a una decisión de un caso. La fuente de la norma usada en la decisión no es relevante porque su validez viene determinada por su uso. En situaciones extremas el Derecho operativo puede ser completamente independiente de cualquier norma jurídica promulgada; es decir, la validez factual es independiente del concepto de validez sistémica. En este sentido validez significa predicción del comportamiento de los operadores jurídicos ${ }^{4}$.

c) Validez axiológica. Según WRÓBLEWSKI, también en este caso pueden darse dos versiones. Según la versión radical las normas jurídicas son válidas si y sólo si son coherentes con determinadas normas extra-jurídicas y/o valoraciones. De acuerdo con la versión moderada, la falta de coherencia es una condición adicional de validez impuesta por la validez sistémica o factual. Tanto en una como en otra versión validez significa aceptabilidad de las normas.

BuLYGIN por su parte, toma como primitivo el término «existencia» del que distingue cuatro conceptos: existencia fáctica, pertenencia, validez y existencia formal 5 . La existencia fáctica se identifica con la vigencia (Ross: una norma es vigente cuando la predicción de que será usada en sentencias judiciales futuras para justificar la decisión del juez es verdadera) o con la «eficacia» (KELSEN: una norma es eficaz si es obedecida por los sujetos jurídicos o aplicada por las autoridades). El análisis más esclarecedor de la existencia fáctica, dice BULYGIN, se debe a HART quien lo analiza en términos de aceptación de la norma como pauta de comportamiento por el grupo social: los miembros del grupo consideran justificadas las conductas conforme a las normas y también consideran justificada la reacción contra aquellos que la violan. Esto implica que consideran que la norma es obligatoria desde el punto de vista interno. Este concepto fáctico de existencia - dice BULYGIN- es descriptivo (decir que una norma existe en una determinada social es afirmar un hecho); admite diferente grados de intensidad (una norma puede existir o estar vigente en mayor o menos grado de-

\footnotetext{
${ }^{4} \mathrm{El}$ concepto de validez factual puede mirar también como destinatario a los ciudadanos. En este sentido ALEXY señala que una norma vale socialmente si es obedecida o en caso de desobediencia se aplica una sanción. Ahora bien, el concepto de obediencia es ambiguo pues uno puede preguntarse si para la obediencia de una norma basta el sólo comportamiento externo o si la obediencia de una norma presupone determinados conocimientos y motivos del actor. En el primer caso hay que destacar tres cosas. De un lado que la validez social (o eficacia social) de una norma es una cuestión de grado (una norma puede ser obedecida y/o aplicada en el ochenta por ciento de los casos en el cincuenta o en el cuarenta por ciento). De otro lado, la validez social es cognoscible recurriendo a dos criterios: el de la obediencia y el de la aplicación de la sanción en caso de desobediencia. Por último, la aplicación de la sanción en caso de desobediencia incluye el ejercicio de la coacción estatalmente organizada en los sistemas jurídicos desarrollados, R. ALEXY, 1997: El concepto y la validez del derecho, p. 88.

5 E. Bulygin, 1991: «Validez y Positivismo», pp.508-511. En el mismo sentido J.R. DE PÁRAMO en J. BETegón, M. Gascón, J.R. De PÁRAmo y L. Prieto, 1997: Lecciones de Teoría del Derecho, pp.160-163.
} 
pendiendo del grado de aceptación) y, es relativo a un determinado grupo social y a un momento temporal.

El segundo sentido de existencia es el de pertenencia. «Se dice que un sistema de normas existe cuando pertenece a un determinado sistema de normas». Y señala que comúnmente los juristas admiten un criterio genético de pertenencia según el cual una norma pertenece a un sistema dado si ha sido creada por la autoridad competente y no ha sido derogada por la misma u otra autoridad del sistema. En este sentido - dicese trata de un concepto descriptivo y relativo (la misma norma puede pertenecer a un sistema y no pertenecer a otro; puede pertenecer a un determinado sistema en un momento y no en otro).

Un tercer sentido de existencia es el de validez. Se trata de un concepto normativo: una norma existe o es válida en este sentido si, y sólo si, es obligatoria. En este sentido, una norma es válida no es afirmar un hecho, sino prescribir la obligación de obedecer la norma en cuestión.

Un cuarto sentido es el de existencia formal. Las normas existen en este sentido, o bien cuando han sido formuladas por alguien (que no necesita ser autoridad jurídica), o bien si son consecuencia lógica de normas formuladas (p. ej., un proyecto de ley que no ha sido aun promulgado: sus normas no existen en los tres significados anteriores, puesto están vigentes, y sin embargo tiene perfecto sentido decir que existen).

VON WRIGHT se refiere a dos sentidos de «validez de una norma». Uno de lo sentidos en que una norma puede decirse que es válida es en el de que existe. Una persona se encuentra con algo que interpreta como una formulación-norma, en un código, por ejemplo. De esto se infiere que ha sido promulgada una norma de tales y tales características y que, por tanto, dicha norma ha existido, al menos durante algún tiempo. Puesto que validez aquí significa existencia — dice— quizá sería mejor no usar la palabra «validez» en absoluto, pues esta palabra también se usa con un significado completamente diferente. Bajo otro significado, la validez de una norma significa que la norma existe y que, además, existe otra norma que permitió a la autoridad de la primera norma emitirla. Aquí validez de una norma «significa la legalidad del acto de emitir esa norma». Así VON WRIGHT concluye señalando que «"valido" y "validez", cuando se aplican a una norma, algunas veces se refieren a la existencia como tal de esa norma, y algunas veces a al legalidad del acto como resultado del cual esta norma cobró existencia» ${ }^{6}$.

En un sentido similar GUASTINI señala que en la literatura se pueden encontrar al menos dos conceptos de validez: $a$ ) en un sentido fuerte de validez, una norma es válida en un sistema jurídico si, y sólo si, cumple todos los criterios de identificación que son específicos de ese sistema. En otras palabras, una norma es válida si, y sólo si, está totalmente libre de cualquier clase de vicio; $b$ ) en un sentido débil de validez, una norma es válida si y sólo si existe, es decir, si ha sido formulada y creada por una autoridad creadora de reglas. En algunos sistemas jurídicos la diferencia entre estos dos conceptos es de gran importancia. En el sistema italiano, una norma aprobada por votación en el Parlamento y promulgada por el presidente de la República cuenta como una «nor-

\footnotetext{
${ }^{6}$ G. H. VON WRIGHT, 1970: Norma y acción, p. 200.
} 
ma válida», en el sentido débil, aunque sea inconsistente con la Constitución. Sin embargo, una norma inconsistente con la Constitución es obviamente «inválida» en el sentido fuerte por el carácter rígido de la Constitución italiana ${ }^{7}$.

\subsection{Concepto de validez}

Como conclusión de lo anterior, puede decirse que el término validez, referido a la validez en el Derecho, es un término que viene siendo utilizado con diversos significados, y en este sentido puede decirse que es ambiguo. Pero esta ambigüedad es en parte artificiosa puesto que puede eliminarse, ya que en la propia teoría del Derecho muchos de los conceptos mencionados bajo el término validez se denominan con otros términos: vigencia, existencia, obligatoriedad, etc., y se trata claramente de conceptos distintos.

Siguiendo las definiciones de VON WRIGHT y GUASTINI antes señaladas, acogeré la siguiente definición de validez: «es válida toda norma, disposición o fuente normativa que haya sido creada de conformidad con todas las normas que disciplinan su creación» ${ }^{8}$.

La validez de las normas está en función de otras normas: las «normas sobre la producción jurídica». Son «normas sobre la producción jurídica» (NPJ) (o «normas de competencia», «reglas que confieren poderes», «normas secundarias») los enunciados jurídicos (disposiciones) que confieren poderes para la producción de nuevos enunciados jurídicos o de proposiciones jurídicas ${ }^{9}$ y también, los enunciados que se refieren a poderes ya concedidos por otros enunciados. En otras palabras, son NPJ aquellos enunciados jurídicos que regulan la creación y aplicación de otros enunciados jurídicos ${ }^{10}$.

Las NPJ establecen de un lado, la definición de cada categoría normativa (o fuente del Derecho) a través de la cual los enunciados jurídicos se incorporan al ordena-

7 R. GUASTINI, 1994: «Invalidity», pp. 224-225.

8 R. GuASTINI, 1999: Distinguiendo, p. 320.

9 Cfr. A. M. PEÑA FreIRE, 2003: Las normas sobre la producción jurídica, p. 144.

10 Teniendo en cuenta los tipos de normas sobre la producción jurídica pueden verse los errores de una definición que podríamos llamar «clásica» sobre la cuestión como la de WRÓBLEWSKI según la cual una norma es válida si cumple las siguientes condiciones: $a$ ) haber sido publicada por una autoridad competente para ello en ese ordenamiento jurídico; $b$ ) no haber sido derogada, y $c$ ) ser coherente con los enunciados válidos del sistema y, caso de no serlo, entonces o pierde su validez según las reglas aceptadas del conflicto de normas, o bien es interpretado de forma que deje de ser incoherente con los enunciados en cuestión. Véase J. WRÓBLEWSKI, 1982: «Tre concetti di validita», pp.587-588, y, 1992: The Judicial Application of Law, p. 77. En sentido similar N. BоввIO, 1958: Teoria della norma giuridica, pp.37-38; 1960: Teoria dell ordinamento giuridico, pp. 119-121; F. OST y M. VAN DE KERCHOVE, 1987: Jalons pour una theorie critique du droit, p. 279.

Respecto de la primera: la publicación y la creación por una autoridad competente son sólo algunas de las NPJ: para ser correcto este requisito debería incluir todas las NPJ. Respecto de la segunda condición, puede considerarse correcta o no según se considere la derogación: como revocación de la validez de la norma; como pérdida de existencia o como limitación del ámbito temporal de validez. Yo me inclino por la tercera, por lo que la derogación no debería incluirse como requisito de validez. Por último, la coherencia no es con carácter general requisito de validez. Lo es cuando entre las normas hay una relación de norma de producción y norma producida y además sean de diferente rango (p. ej., ley y reglamento de desarrollo de dicha ley) o del mismo (ley de bases y decreto legislativo ), pero no lo es cuando existen dos normas en el ordenamiento entre las cuales no se da esa relación de norma de producción y norma producida (por ejemplo puede haber dos normas contradictorias en el código civil, producidas ambas dos dentro de los márgenes de las NPJ y ser ambas válidas). 
miento y, de otro, el régimen jurídico de cada categoría normativa señalado qué relaciones va a tener cada fuente del derecho y cada enunciado en ella incluido con todas las demás. Haciéndome eco de una tipología ampliamente asumida, las NPJ incluyen las siguientes clases ${ }^{11}$ :

a) Normas que confieren poderes. Se trata de enunciados que confieren a un determinado sujeto u órgano del Estado un poder normativo, o sea, la competencia para crear disposiciones. Este tipo de enunciados no suelen realizar una atribución genérica para crear enunciados jurídicos, sino para crear una específica fuente de derecho con un «nombre propio» (ley ordinaria, ley orgánica...). Los enunciados jurídicos no tienen «autonomía», sino que todo enunciado jurídico se distingue por la pertenencia a una determinada fuente. A su vez, toda fuente se distingue por su nombre y su régimen jurídico; régimen que incluye cuestiones como la fuerza, el campo de aplicación, etcétera.

Este tipo de enunciados tiene dos vertientes. De un lado, son normas de conducta y en particular normas permisivas (si bien en determinados casos son normas obligatorias). Por otro lado, son definiciones del tipo de fuente del derecho sobre la que versan. La atribución de una competencia normativa no puede presumirse, sino que debe ser consecuencia de una clara habilitación, lo que conlleva que ninguna otra autoridad dotada de poder normativo puede elaborar documentos pertenecientes a ese tipo de fuente.

b) Normas de procedimiento. Son normas que regulan el procedimiento para crear las fuentes de Derecho o, más correctamente, que establecen un procedimiento para la elaboración de cada tipo de fuente o disposición. También este tipo de enunciados presentan un doble aspecto. De un lado son normas de conducta, en particular, mandatos, dirigidos a las autoridades normativas y relativas a un tipo de conducta consistente en crear enunciados jurídicos. De otro, constituyen una definición del tipo de fuente a la que refieren.

c) Normas que circunscriben el ámbito de poder conferido, determinando qué materias se pueden regular con el poder en cuestión. Se trata de normas relativas al ámbito material de una determinada fuente, es decir, el conjunto de materias sobre las que la fuente puede promulgar enunciados jurídicos. Son ejemplo de este tipo de normas, tanto aquellas que enumeran exhaustivamente las materias que puede regular una determinada fuente de derecho, como las que lo indican de manera más genérica.

d) Normas que reservan una determinada materia a una determinada fuente, de manera que: i) ninguna otra fuente está habilitada para regular esa materia, y ii) la fuente en favor de la que se establece la reserva no está autorizada para delegar la regulación de la materia en cuestión a otras fuentes. Estas normas incluyen: un permiso para que el órgano competente regule esa materia, una probibición a cualquier órgano para regular esa materia por medio de otra fuente y, una probibición al órgano competente de delegar a otras fuentes para regular esa materia.

11 R. GuAstini, 1998: Teoria e dogmatica delle fonti, pp. 45-353; 1999: Distinguiendo, pp. 309-310; 1995: Il giudic e la legge, cap. V. Cfr. A. M. PEÑA FrEIRE, 2003: Las normas sobre la producción jurídica, pp. 119-154; J. FERRER BELTRÁN, 2000: Las normas de competencia; R. HERNÁNDEZ MARÍN, 2002: Introducción a la teoría de la norma jurídica, pp.343-370. 
e) Normas relativas al contenido de la regulación futura. Se trata de límites sustanciales, prohibiendo a una determinada fuente crear normas con un determinado contenido o imponiendo que estas tengan un contenido determinado. Se trata por tanto de prohibiciones (u obligaciones) impuestas al órgano al que se refieren ${ }^{12}$.

\subsection{Juicio de validez}

2.3.1. Los juicios de validez son enunciados del tipo «La norma $\mathrm{N}$ es una norma válida/inválida». Aquí la expresión «la norma $N »$, puede tener diversos sentidos en función de los significados de «norma jurídica»: acto lingüístico, disposición y norma.

a) Acto lingüístico. El enunciado «Es válida/inválida la norma $N »$ puede referirse a la relación entre un determinado comportamiento (el acto lingüístico de legislar) y una norma o conjunto de normas de producción.

Legislar es un acto institucional o, más bien, una secuencia de actos institucionales gobernados por normas jurídicas constitutivas (es decir, la noción de legislación no puede ser definida sin mencionar en el definiens las normas en cuestión) ${ }^{13}$. Estas normas constitutivas son «normas sobre la producción jurídica» que regulan la producción de leyes. Las NPJ confieren al acto «bruto» de prescribir (realizado por determinados sujetos conforme a ciertos procedimientos) la etiqueta institucional. Legislar consiste en la realización de una secuencia de actos y comportamientos que dan lugar a la producción de un texto normativos; actos que, en su conjunto, constituyen el procedimiento legislativo.

b) Disposición y norma. El enunciado «Es válida la norma $N »$ puede versar sobre la relación entre la disposición y la norma, y en este sentido una disposición es válida si expresa al menos una norma válida.

Partiendo de esta distinción, GUASTINI distingue entre validez formal y material: la validez formal de una disposición depende de la validez del acto normativo del cual ha sido producida; mientras la validez material de una disposición depende de la validez de las normas que de la misma pueden obtenerse mediante interpretación. Se dice que una disposición es válida desde el punto de vista formal cuando ha sido producida mediante un acto normativo formalmente válido. En otros términos, las condiciones de validez formal de una disposición se resuelven en las condiciones de validez del acto normativo por medio del cual dicha disposición ha sido producida (conformidad a las metanormas sobre la producción jurídica, concretamente las de

${ }^{12}$ Los límites pueden clasificarse en dos categorías: directos e indirectos. Los primeros son todos los enunciados que predeterminan positivamente (imponiendo obligaciones, como lo hacen por ejemplo los enunciados programáticos de la Constitución imponiendo promover determinados fines económicos o sociales) o negativamente (imponiendo prohibiciones, p.ej., la prohibición de discriminación del art.14 de la Constitución) el contenido de la legislación. Son límites indirectos los que derivan de la combinación de dos tipos de enunciados: i) por un lado, los enunciados con los cuales una determinada fuente regula directamente cierto supuesto de hecho (p. ej., confiriendo derechos a los ciudadanos); ii) por otro, los enunciados con los cuales la fuente en cuestión establece su propia superioridad jerárquica sobre otras fuentes., p. ej., la Constitución sobre cualquier tipo de ley). En virtud de este tipo de relación el enunciado subordinado no puede contener enunciados que contradigan otros incluidos en la fuente superior.

${ }^{13}$ R. GuASTINI, 1999: Distinguiendo, p.307. 
competencia y de procedimiento, no de la disposición misma sino, del acto normativo). Una disposición normativa es válida desde el punto de vista material cuando las normas (explícitas) que de la misma pueden obtenerse son materialmente válidas. Más precisamente, una disposición es válida si expresa al menos una norma materialmente válida; pero una disposición es inválida si todas las normas que ella expresa son materialmente válidas ${ }^{14}$.

Considero que resulta más clarificador referir la validez al conjunto de NPJ, sin distinguir entre validez formal y marial en función del tipo de NPJ, pues la validez se define por el cumplimiento de todas las NPJ. Es obvio entre las NPJ hay algunas unas con mayor facilidad interpretativa que otras, pero ello no da lugar a dos tipos de validez.

2.3.2. Un juicio de validez es un enunciado interpretativo en el cual están involucrados los siguientes enunciados:

EO) Enunciado objeto del juicio de validez: es un enunciado que pertenece a un texto jurídico, y cuya validez es examinada.

EP) Enunciado (o enunciados) que constituye el parámetro del juicio de validez: son las normas de producción jurídica relativas a EO, más las normas supremas (éstas, aunque no estén directamente relacionadas con EP constituyen también un parámetro de validez).

ER) Enunciado resultado del juicio de validez; es un enunciado interpretativo que, a diferencia de los anteriores, pertenece al lenguaje de los juristas o jueces y es un metalenguaje respecto a los dos enunciados anteriores (EO y EP).

El carácter interpretativo de ER se hace patente si se tienen en cuenta las NPJ. Estas suelen contener requisitos formales (de procedimiento) y materiales (de contenido). Ambos son requisitos de validez: la diferencia entre unos y otros radica en la mayor o menor dificultad interpretativa, pero todos ellos constituyen requisitos de validez. Como ha señalado JORI «de hecho las llamadas normas formales (por lo general normas de competencia o de procedimiento) son casi siempre semánticamente más precisas y rigurosas que las otras», pero manifiestan «los mismos problemas de indeterminación semántica, discrecionalidad interpretativa y referencias a parámetros valorativos» ${ }^{15}$.

14 La relación entre los criterios de validez formal y material es la siguiente. La validez formal es una condición ni suficiente ni necesaria de la validez material. De un lado, la validez formal no es condición suficiente de la validez de una norma. En otros términos, una norma explícita puede ser válida desde el punto de vista formal e inválida desde el punto de vista material (p.ej., el legislador puede produce una norma valida desde el punto de vista del proceso de formación del derecho, pero a su vez ser incompatible con la Constitución; con lo cual será materialmente inválida (es decir, inválida en razón de su contenido). En algunos casos la invalidez de una norma afecta a la disposición invalidando aquélla. De otro lado, la validez formal tampoco es condición necesaria de la validez material de una norma. Una norma implícita (por tanto, inválida bajo el punto de vista formal) puede ser válida desde el punto de vista material. Los juristas consideran (materialmente) válidas muchas normas que no han sido formuladas por el legislador, pero que en su opinión pueden ser obtenidas de alguna norma explícita o de la combinación de normas explícitas. Entre los dos criterios de validez se da la siguiente relación: en caso de conflicto entre ellos el criterio material prevalece sobre el formal. En otros términos: a) si una norma (explicita) es válida desde el punto de vista formal pero, al mismo tiempo, invalida desde el punto de vista material, es considerada tout court inválida; $b$ ) si una norma (implícita) es inválida desde el punto de vista formal pero, al mismo tiempo, valida desde el punto de vista material, es considerada tout court válida. La validez formal es una validez «débil» mientras la validez material es «fuerte», R. GUASTINI, 1989: «Concetti e criteri di validità», p. 877 .

15 M. JORI, 1993: «La cicala e la formica», p. 84. 
Por otro lado, ha estado (o está) arraigada la idea de que la validez de los enunciados jurídicos es una característica meramente formal. Así para RAz, la validez jurídica es establecida no por argumentos sobre su valor y justificación, sino mostrando que la norma se conforma a criterios de validez establecidos por algunas otras normas del sistema: las reglas de reconocimiento, «tales criterios se refieren normalmente a la forma en que la norma fue legislada o establecida por la autoridad judicial $»^{16}$. Asimismo, cuando HART explica la variedad de criterios de pertenencia al sistema jurídico que puede contener una regla de reconocimiento, sólo alude a criterios formales, como «la referencia a un texto revestido de autoridad; a una sanción legislativa; a la práctica consuetudinaria; a las declaraciones generales de personas especificadas, o a las decisiones judiciales pasadas, dictadas en casos particulares»; y considera Derecho «todas las reglas que son válidas según los criterios formales de un sistema de reglas primarias y secundarias» ${ }^{17}$. Y MACCORMICK ha señalado que «es común tomar como una característica definitoria del positivismo que todo positivista genuino mantiene que todas las normas que son normas jurídicas lo son porque pertenecen a un particular ordenamiento jurídico, y que pertenecen al sistema porque satisfacen criterios formales de reconocimiento» ${ }^{18}$. En la doctrina constitucional española, hay opiniones, como la de REQUEJO PAGÉS según la cual: «una norma es válida en la medida en que su generación se ha verificado con arreglo a los procedimientos de producción normativa instaurados en el sistema del que forma parte. Es éste un concepto de validez puramente procedimental, en el que se hace abstracción de consideraciones de índole material y axiológica, en la idea de que, en último término, un sistema normativo no es más que un entramado de procedimientos a cuyo través puede dotarse a las formas jurídicas de cualquier contenido. Las supuestas condiciones materiales de validez no son otra cosa, en puridad, que simples condiciones de procedimiento» ${ }^{19}$.

Este privilegio de la vertiente formal de la validez resulta paradójica cuando, según hemos visto, las normas de producción jurídica que establecen los criterios de validez imponen no sólo límites formales, sino también materiales, esto es de contenido. La idea opuesta la expresa perfectamente FERRAJOLI cuando dice que «La definición teórica de "derecho válido" — con arreglo al principio metacientífico del positivismo jurídico expresado por nuestra tesis acerca de la separación entre Derecho y moral- sigue siendo por tanto ideológicamente neutral: es válida, aunque sea injusta, cualquier norma o prescripción que sea conforme a las normas acerca de su producción. Lo que ocurre es que entre las normas acerca de la producción de normas el moderno estado constitucional de derecho ha incluido múltiples principios ético-políticos o de justicia, que imponen valoraciones ético-políticas de las normas producidas y actúan como parámetros o criterios de legitimidad y de ilegitimidad no ya externos o iusnaturalistas,

${ }_{16}^{16}$ J. RAZ, 1982: La autoridad del derecho, p. 193

17 H.L. A. HART, 1998: The Concept of Law, pp. 101 y 109.

18 N. MACCORMICK, 1978: Legal Reasoning and Legal Theory, p. 61 (la cursiva es mía).

${ }^{19}$ J.L. ReQueJo PAGÉs, 1998: Las normas preconstitucionales y el mito del poder constituyente, pp.26-27. Si bien luego dice: «los únicos límites materiales jurídicamente admisibles son los representados por las decisiones materiales formalizadas en normas que condicionan la validez de normas inferiores y cuya revisión está sometida a especiales dificultades de procedimiento. Tal es, señaladamente, el caso de las normas constitucionales relativas a los derechos fundamentales, en las que se formalizan límites de contenido que evidencian su condición última de de límites procedimentales a poco que se repare en el hecho de que es posible su revisión a través de la reforma constitucional». 
sino internos o iuspositivistas... La especificidad del moderno Estado constitucional de derecho reside precisamente en el hecho de que las condiciones de validez establecidas por sus leyes fundamentales incorporan no sólo requisitos de regularidad formal, sino también condiciones de justicia sustancial. Estos rasgos sustanciales de la validez inexplicablemente ignorados por la mayor parte de las definiciones iuspositivistas de "Derecho válido", ya sean normativistas o realistas, tienen una relevancia bastante mayor que la de los meramente formales $»^{20}$.

2.3.3. En cuanto al objeto del juicio de validez al que me he referido antes, hay que decir el juicio de validez no puede predicarse de todas las disposiciones de un orden jurídico, pues quedan excluidas del mismo las normas supremas; la razón es obvia: el concepto mismo de norma suprema impide que pueda ser sometida a un juicio de validez en el ordenamiento.

Una situación diferente se da con las normas persistentes o receptadas ${ }^{21}$, respecto de las cuales sólo se les exige el cumplimiento de parte de las NPJ.

Para explicar el concepto de normas receptadas hay que partir de la distinción entre «sistema jurídico», es decir, el «conjunto de normas agrupadas en virtud de algún criterio específico» $\mathrm{y}$ «orden jurídico», esto es, la sucesión de estos conjuntos que por algún factor o circunstancia se nos muestran como una secuencia unitaria o como dotados de una misma identidad ${ }^{22}$. En otras palabras: la expresión «sistema jurídico» se emplea para referirse a conjuntos de normas asociados a un tiempo $t$ determinado, mientras que «orden jurídico» hace referencia a una secuencia de sistemas jurídicos. Una norma jurídica pertenece a un sistema jurídico y un sistema jurídico pertenece a un orden jurídico. Así, para cada momento determinado hay un conjunto de normas que conforman un sistema jurídico. Además distintos sistemas jurídicos asociados a distintos momentos pertenecen al mismo orden jurídico ${ }^{23}$.

Desde el punto de vista dinámico, un orden jurídico sucede a uno anterior y también es sucedido por otro posterior. Si referimos este proceso a algún momento histórico o caso real que nítidamente interpretemos como la sustitución de un orden jurídico por otro, puede comprobarse que tras la desaparición de un orden jurídico y sustitución por otro, no se hace tabula rasa de todas las normas jurídicas pertenecientes al último sistema del orden anterior; por lo general, la mayoría de ellas seguirá siendo considerada como parte del Derecho de la unidad política de que se trate. La fundación en un momento histórico de un nuevo orden jurídico no implica en términos bistóricos la extinción o derogación de todas y cada una de las normas que pertenecían a su último sistema, sino que «las normas anteriores a la promulgación de una nueva constitución, elaborada al margen de las reglas anteriores a su aparición (...), siguen usándose después de la promulgación de la nueva constitución para atribuir obligaciones y derechos a los individuos por los tribunales y por los operadores jurídicos en ge-

20 L. FERRAJOLI, 1995: Derecho y razón, p. 358.

${ }^{21}$ Cfr. J.M. VillajosAnA, 1998: «Sobre recepción de normas» y, 1998: «Dinámica de sistemas y persistencia de normas jurídicas», pp.49-64.

${ }^{22}$ Es la diferencia que hace E. BuLYGIN, 1991: «Tiempo y validez», p. 197; 1991: «Algunas consideraciones sobre los sistemas jurídicos», p.260, entre tiempo interno y tiempo externo. $C f r$. sobre esta distinción J.J. MORESO y E. NAVARRO, 1993: Orden jurídico y sistema jurídico, pp.41-44.

${ }^{23}$ J.M. Villajosana, 1998: «Dinámica de sistemas y persistencia de normas jurídicas», p. 52 
neral $\gg^{24}$. Por esto, en los casos de sustitución de un orden jurídico por otro, por lo general desaparecen algunas normas importantes, pero las demás prolongan su vigencia en los sistemas del nuevo orden. A las normas que subsisten de un orden a otro se las califica como «normas persistentes»o «normas receptadas» ${ }^{25}$. Por tanto, las normas receptadas no tienen que cumplir todas las NPJ del ordenamiento, sino únicamente las normas relativas al contenido; lo que da lugar a lo que ViLLAJOSANA llama criterio sustantivo de pertenencia y que formula como sigue: «Una norma $N$ pertenece al último sistema jurídico del orden jurídico O1 pertenece a un sistema jurídico del orden jurídico $\mathrm{O} 2$ del mismo orden estatal si su contenido es compatible con las normas del primer sistema jurídico de $\mathrm{O} 2 »^{26}$.

\section{EXISTENCIA}

\subsection{Planteamiento}

La cuestión de la existencia de las normas se plantea de manera radicalmente diferente a la de la validez. La validez, como la vigencia, la aplicabilidad, la eficacia, etc. son propiedades o características que las normas pueden tener o no. La existencia, por el contrario, no es una propiedad; la existencia es diferente de las propiedades, «la existencia no añade nada a la cosa, no es una propiedad más, sino que es simplemente la posición de una cosa determinada con todas sus propiedades delante de un sujeto ${ }^{27}$.

Como señala LÓPEZ HERNÁNDEZ ${ }^{28}$, «para decir que una cosa existe, primero, tiene que poder ser descrita como una entidad caracterizada por la posesión de algunas propiedades que le dotan de una identidad y que la hacen identificable a través de los mismos, y segundo, esa entidad así caracterizada tiene que aparecer delante de un sujeto cognoscente». La forma de aparición de la cosa ante el sujeto es lo que llamamos su modo de existencia, que será diferente según el tipo de entidad de que se trate: si es una entidad factual deberá mostrarse ante el sujeto empíricamente, si es una entidad ideal, deberá mostrarse de una manera abstracta.

Dicho de otra manera, el verbo «ser» puede emplearse en dos sentido: 1) en sentido copulativo $(x$ es $P$ ), y entonces actúa para atribuir propiedades a un objeto; 2 ) en sentido existencial ( $x$ existe), y entonces actúa para afirmar la presencia de un objeto cualquiera con todas sus propiedades ante un sujeto cognoscente. Ambos usos son independientes y en ninguno de los casos «ser» figura como predicado, o sea, no es una propiedad: en el primer caso enlaza predicados a sujetos y en el segundo caso solamente afirma la existencia de objetos individuales de los que, en enunciados distintos, se pueden haber predicado algunas propiedades.

${ }^{24}$ J.J. MORESO, 1997: La indeterminación del derecho y la interpretación de la Constitución, p. 159.

25 A. M. PeÑa FreIRE, 2001: «Los sistemas jurídicos y la aplicabilidad de sus normas», p. 207. Véase J. M. VILLAJOSANA, 1998: «Sobre recepción de normas».

26 J.M. VILLAJOSANA, 1998: «Dinámica de sistemas y persistencia de normas jurídicas», p. 63.

27 Esta idea me fue sugerida por J. M. VILlajosanA. Vid. J. LÓPEZ HERNÁNDEZ, 2004: «Existencia, validez y nulidad de las normas jurídicas», Anales de Derecho, n. ${ }^{\circ} 22$, p. 103.

${ }^{28}$ J. LÓPEZ HERNÁNDEZ, 2004: «Existencia, validez y nulidad de las normas jurídicas», Anales de Derecho, n. ${ }^{\circ} 22$, p. 104. 
Partiendo de este planteamiento, el tema de la existencia de las normas jurídicas es una cuestión complemente distinta de la de la validez: se trata de saber si existen normas jurídicas (cosa que se da por supuesta); y de saber bajo qué condiciones o circunstancias existen tales normas ${ }^{29}$.

Por tanto, la cuestión de la existencia de las disposiciones se plantea en los siguientes términos: cuándo una norma jurídica existe o, lo que es lo mismo, cuándo estamos ante una norma jurídica. Para ser más precisa, voy a me voy a referir únicamente a la existencia de las disposiciones.

\subsection{Diferentes nociones de existencia}

\subsubsection{Existencia formal}

Una primera respuesta a la pregunta cuándo existe una disposición, es hacerlo en términos de existencia formal; se trata de probar la existencia de una disposición desde el punto de vista del derecho es decir, en función de factores y criterios de carácter jurídico.

Comencemos por algunas definiciones de existencia que parten de esta idea:

Def. 1: «Que una ley existe o está vigente significa sólo que está contenida en un acto legislativo no anulado y puede por consiguiente ser aplicada mediante decisiones válidas respecto a ella» ${ }^{30}$.

Def. 2: «Uno de los sentidos en que una norma puede decirse que es válida es en el de que existe. Una persona se encuentra con algo que interpreta como una formulación-norma, en un tablero de anuncios o en un libro de estatutos, por ejemplo. De esto se infiere que ha sido promulgada una norma de tales y tales características y que, por tanto, dicha norma ha existido, al menos durante algún tiempo» ${ }^{31}$.

Def. 3: Las reglas de competencia recogen en su antecedente las condiciones necesarias y suficientes para la existencia de las fuente-acto. Dentro de estas condiciones podemos distinguir entre la competencia formal, la material, la personal, la territorial y

${ }^{29}$ Un concepto próximo al de existencia (y en muchas ocasiones identificado con él) es el de vigencia. La vigencia de las normas puede definirse como «su capacidad regulativa, su aptitud para regular las situaciones que caigan bajo su condición de aplicación; su aplicabilidad en definitiva. Esto significa que una norma regula cuántas situaciones se produzcan mientras la misma está en vigor, y, en consecuencia, que la norma podrá ser usada por los jueces para justificar su decisión cuando tengan que resolver conflictos referentes a dichas decisiones. Cfr. M. Gascón en J. Betegón, M. Gascón, J. R. De PÁramo y L. Prieto, 1997: Lecciones de Teoría del Derecho, p. 249. L. FERRAJOLI, 1995: Derecho y razón, pp. 873-874, identifica existencia con vigencia. Pues bien, existencia y vigencia son conceptos distintos puesto que la vigencia es una propiedad de la norma: un a norma puede existir, es decir ser norma jurídica, y no estar vigente.

30 L. FerRAJOLI, 1995: Derecho y razón, p. 873. Sobre la noción de vigencia, véase J. Delgado PINTO, 1990: «Sobre la vigencia y validez de las normas», pp. 101-167.

31 G. H. von Wright, 1970: Normas y Acción, p. 200. Cfr. C. E. Alchourrón y E. Bulygin, 1979: Sobre la existencia de las normas jurídicas, p. 43, quienes definen la existencia en términos de promulgación seguidamente decir que aunque no es un concepto de existencia válido para dar cuenta de la existencia temporal de las normas jurídicas. Y más adelante (p.74) señalan que sólo de normas promulgadas cabe decir que son válidas o inválidas. En otras palabras, la validez y la invalidez sólo puede predicarse respecto de normas existentes (en el sentido de promulgación). 
otras condiciones que generalmente suelen referirse a la oportunidad. «Del hecho de que se configuren como condiciones necesarias del resultado institucional, de la fuente-acto, se sigue que la no satisfacción de cualquiera de ellas hace que no se produzca el resultado institucional, es decir que no exista la fuente acto» ${ }^{32}$.

Como se desprende de estas definiciones, estos criterios jurídicos no son otros que las NPJ. La pregunta siguiente es qué NPJ son necesarias para que exista una disposición; pues parece claro que no es necesario el cumplimiento de todas las NPJ para la existencia de las disposiciones, sino sólo para su validez.

A esta cuestión se han dado varias respuestas. Para un grupo de autores la cuestión depende del cumplimiento de determinadas NPJ. Para GUASTINI por ejemplo, una fuente de derecho es no sólo inválida sino no-existente si es producida por un órgano que no tiene competencia para producir esa clase de fuente. Si se infringen las reglas de procedimiento, la cosa es dudosa. A veces una fuente de derecho producida infringiendo una regla de procedimiento es existente, aunque inválida. A veces sin embargo es considerada no-existente. «Como norma general la infracción de una única regla de procedimiento simplemente implica invalidez, mientras que la infracción de todas las reglas de procedimiento implica no-existencia. Pero los conceptos de invalidez e inexistencia tienen textura abierta. Es imposible afirmar cuantas reglas de procedimiento se deben violar para alcanzar la no-existencia» ${ }^{33}$. No se puede trazar una línea clara de demarcación entre invalidez e inexistencia..., no es posible decir con precisión a qué y a cuántas NPJ debe ser conforme una norma para superar el umbral de la existencia sin alcanzar la plena validez ${ }^{34}$. En otro lugar dice que si bien es difícil determinar cuáles son los requisitos para que un enunciado se considere existente, puede decirse que son los siguientes: que haya sido creado por un órgano prima facie competente y que se hayan respetado algunas normas procedimentales (aunque es difícil determinar cuales sean estas exactamente ${ }^{35}$.

PEÑA FrEIRE llega a la siguiente conclusión: las NPJ que confieren poder al órgano $\mathrm{O}$ para crear normas de la clase $\mathrm{N}$, más las normas de procedimiento que resulten constitutivas del órgano $\mathrm{O}$ determinan la existencia. Las demás normas, es decir, las normas de procedimiento, las normas de competencia, las de reserva y las de contenido, no determinan la existencia de las normas, únicamente su validez. En un intento

32 J. Agulló, 2000: Teoría general de las fuentes del derecho, p. 81. En pp. 67-68 se refiere a las fuentesacto como sigue: «la legislación, tomada en su sentido más amplio, es el ejemplo paradigmático que guiará nuestra exposición sobre las fuentes-acto y sobre las normas provenientes de autoridades políticas». Señala también que las fuentes-acto presentan cuatro elementos constitutivos: $a$ ) la autoridad normativa, esto es, un sujeto investido de poder (competencia) para crear Derecho; $b$ ) el acto normativo, es decir el acto o secuencia de actos productores de normas; $c$ ) el texto o documento normativo: el resultado de lo anterior es un documento, un conjunto de enunciados que cumplen una función prescriptiva; enunciados que componen una disposición, y d) las normas, esto es, el contenido significativo que resulta de la interpretación del documento normativo o disposición.

33 R. Guastini, 1994: «Invalidity», pp. 222-223. En Teoria e dogmatica delle fonti, 1998, p. 147, señala: «Una norma "existe" en un ordenamiento jurídico —o bien pertenece al mismo- si, y sólo si, ha sido no sólo formulada (como, p. ej., las normas de un proyecto de ley), sino además "puesta" o sea discutida y emanada (además de publicada), de acuerdo con al menos algunas de las normas sobre la producción jurídica propias del ordenamiento».

34 R. GUASTINI, 1994: «Invalidity», pp.179, 183. En el mismo sentido R. GUASTINI, 1999: Distinguiendo, p.321.

35 R. GuASTINI, 1999: Distinguiendo, p.320. 
de precisar más la respuesta señala lo siguiente: «Diremos que una norma $\mathrm{N}$ existe como tal cuando el órgano que la creó tenía poder para hacerlo o dicho de otro modo, una decisión $\mathrm{D}$ - hecho bruto- cuenta como norma de la clase $\mathrm{N}$-hecho institucional- cuando el órgano $\mathrm{O}$ que la produjo tenía conferido poder para crear normas de la clase $\mathrm{N}^{36}$.

GASCÓN, por su parte, después de plantear los problemas que presenta distinguir entre validez y nulidad en base al tipo de NPJ que se infrinja, parte de que la elección del criterio que trace la diferencia entre validez y invalidez debe ser coherente con la función que la inexistencia está destinada a cumplir, función que consiste en establecer un particular régimen de invalidación orientado a reaccionar de un modo expeditivo frente a aquellos actos o normas que resultan más groseramente irregulares, y que la inexistencia así construida no debe dejar sin eficacia al régimen general de invalidación establecido en el sistema. De esta forma llega a la conclusión de que «parece que tan sólo cabe pensar como criterio de la inexistencia en la manifiesta e indubitada inconstitucionalidad, dado que éste es un elemento que justifica la inmediata ineficacia (inaplicación) de la norma en el sistema y que, al mismo tiempo, no cancela el régimen general de invalidación establecido»... «En suma, diremos que una norma es inexistente cuando carece en absoluto de una apariencia de validez; es decir, cuando su invalidez sea manifiesta, evidente y fácilmente recognoscible; cuando no hay dudas de que, llegado el caso, la norma será declarada inválida por el órgano competente». «Llamamos inexistencia a los vicios que prevemos determinarán la invalidez con toda seguridad o, lo que es igual, a la manifiesta e indubitada inconstitucionalidad $»^{37}$. Por otra parte, para GASCÓN el juicio de inexistencia, como el de validez «es un juicio descriptivo destinado a constatar si determinada norma reúne las condiciones de pertenencia establecidas en el sistema». Pero además presenta una dimensión predictiva «porque requiere aventurar que ese vicio es de tal naturaleza o magnitud que, con toda seguridad, conducirá a la declaración de invalidez» ${ }^{38}$. Y en un sentido similar se pronuncia Aguiló, para quien hay ejemplos en los que el resultado institucional de producción de la fuente-acto no se produce, es decir no existe; y señala como ejemplo los siguientes: El Parlamento español (fuera de su ámbito formal de competencia) trata de producir el resultado «ley autonómica»; el Parlament de Catalunya (dentro de su competencia formal y fuera de su competencia territorial) trata de «ordenar el territorio andaluz», el Parlament de Catalunya (dentro de su competencia formal y material y fuera de su competencia personal) trata de extender el régimen económico-matrimonial de la separación de bienes a aquellos que no están sujetos al Derecho foral catalán, etc. En todos estos casos —dice Aguiló- parece claro que las autoridades en cuestión fracasarían en su pretensión de producir los resultados institucionales que llamamos fuentes-acto. El efecto jurídico de estos intentos sería la inexistencia del resultado institucional ${ }^{39}$.

Para otros autores el elemento que determina la existencia es la promulgación o publicación. Así AlCHOURRÓn y BuLYGIN, indican que para explicar la existencia de las

36 A. M. PEÑA FreIRE, 1999: «Reglas de competencia y existencia de las normas jurídicas», pp. 400-401.

37 M. GASCÓN ABELLÁN, 1997: «Sentido y alcance de algunas distinciones sobre la invalidez de las leyes», pp. $149-150$.

38 Ibídem, pp. 150-151.

39 J. AguiLÓ, 2000: Teoría general de las fuentes del derecho, p. 81. 
normas jurídicas basta el acto de prescribir, es decir, la promulgación que hará nacer la relación normativa sin necesidad de recepción por parte de sus destinatarios ${ }^{40}$. Y HERNÁNDEZ MARín afirma que son jurídicos todos aquellos enunciados que aparecen contenidos en un texto o documento que, a su vez, tenga la consideración de cuerpo jurídico; y señala que «para que un enunciado añada a su existencia factual y lingüística la existencia jurídica, es condición necesaria y suficiente que el documento en el que se halla dicho enunciado se convierta en un cuerpo jurídico», y que «un cuerpo jurídico es un cuerpo legal que ha sido publicado» ${ }^{41}$.

Considero que éste es el criterio es el más adecuado como criterio de existencia de las disposiciones. Sin embargo algunos autores añaden un adjetivo: que se trate de un acto serio de promulgación: «todo acto (serio) de promulgar una norma da lugar a la existencia de una norma. Lo único que queda excluido son los actos no serios (por ejemplo, bromas, prescripciones realizadas por un actor en un escenario, etc.) $\rangle^{42}$. Pero los ejemplos citados de actos no serios de promulgación, ni siquiera pueden considerarse actos de promulgación o mejor, de publicación en un diario oficial. Lo mismo ocurre en el caso de enunciados que, no obstante la inserción de un texto en un diario oficial, le falta cualquier apariencia de texto jurídico (p. ej., porque no se corresponde con ninguna fuente de derecho de ese ordenamiento, o porque el sujeto que lo publica es una persona que nada tiene que ver son los órganos competentes, etc.).

\subsubsection{Existencia convencional}

Una segunda respuesta es aquella que entiende la existencia de una disposición como existencia convencional. En el sentido de existencia convencional se dice que «el enunciado que predica la validez constitutiva (o existencia) de una entidad A en el sistema $\mathrm{S}$, será válido siempre y cuando concurran determinados hechos que nos permitan afirmar que $A$ es una entidad convencional en $S »^{{ }^{43}}$. La pregunta es: ¿en qué consiste tales hechos? ${ }^{\text {44 }}$. La respuesta es que se trata de «hechos o entidades convencionales».

De manera sintética puede decirse que los dos rasgos distintivos de una entidad convencional son los siguientes. En primer lugar, la creencia mutua entre los miembros de la comunidad S en la entidad A. En segundo lugar, que la existencia de tal creencia genera entre los miembros de $\mathrm{S}$ alguna razón para la acción que, de otra forma, no ten-

${ }^{40}$ C. E. AlChOURRÓN y E. Bulygin, 1979: Sobre la existencia de las normas jurídicas, p. 37.

${ }^{41}$ R. HERNÁNDEZ MARÍN, 2002: Introducción a la teoría de la norma jurídica, p. 444.

42 C.E. AlChOURRÓn y E. BulYgin, 1979: Sobre la existencia de las normas jurídicas, p. 37. Cfr. también J. J. MoRESO, 1997: Normas jurídicas y estructura del derecho, pp. 29 y 86, que se refiere también a la promulgación de las normas «exitosamente», aunque creo que se refiere más a la vigencia, que la existencia puesto que señala «Una norma $\mathrm{N}$ es vigente si y sólo si ha sido promulgada exitosamenete. Determinar las condiciones en que una norma $\mathrm{N}$ es exitosamente promulgada es delimitar las condiciones en que una norma (jurídica) exista socialmente».

43 A. RÓDENAS, 2006: «Sobre la validez jurídica: entre la validez y la convención», p. 259.

${ }^{44}$ Una norma existe cuando ciertos actos, hechos o documentos son considerados como normas jurídicas por los miembros de un grupo social, ya se trate de particulares o de autoridades. Esta forma de existencia da cuenta de un lado, de la naturaleza social del Derecho y de otro, de que los procedimientos de identificación del Derecho y de las normas no son ajenos a las creencias y actitudes de los miembros respecto de lo que el derecho es A. M. PeÑA FreIRE, 1999: «Reglas de competencia y existencia de las normas jurídicas», p. 382. 
drían. En cuanto al primer requisito, tratándose de entidades convencionales de carácter jurídico la comunidad relevante es la de los operadores jurídicos: basta con que una parte suficientemente extensa de ellos crean en la existencia de la entidad A y que los sujetos tengan una percepción del carácter compartido de la creencia. El segundo requisito (y teniendo en cuenta que la comunidad de referencia es la de los operadores jurídicos) se concreta en que al menos en algunos casos la creencia en la existencia de la entidad jurídica forme parte del conjunto de razones que opera en el trasfondo de las resoluciones de los operadores jurídicos ${ }^{45}$.

Esta respuesta está basada en las ideas de SEARLE y LAGERSPETZ de los «hechos institucionales» (o «hechos dependiente de la creencia») cuya idea fundamental es que hay «hechos» cuya subsistencia depende de la creencia de que subsistan: se da el caso que $p$ sólo si se cree que se da el caso que $p$ (p.ej., algo es dinero sólo si se cree que es dinero, o un determinado conjunto de eventos constituye un matrimonio sólo si se cree que constituye un matrimonio). Los hechos sociales que cumplen esta condición se denominan «hechos dependientes de la creencia» o «hechos institucionales». Este tipo de hechos (sociales) son elementos fundamentales de la realidad social; aun siendo hechos de tipo peculiar, pueden considerarse existentes o no existentes, pueden ser objeto de afirmaciones de hecho, de carácter descriptivo susceptibles de verdad o falsedad. Los hechos institucionales se caracterizan por una relación de circularidad entre el ser y el creer. En general, en relación a todo tipo de hechos es posible creer de modo verdadero que $p$ sólo si se da el caso que $p$. En el caso de los hechos institucionales, sin embargo, vale también la relación inversa: si $p$ es un hecho institucional es posible que se de el caso que $p$ sólo si se cree que $p^{46}$.

Según SEARLE podemos distinguir entre «hechos brutos» (independientes de los estados mentales y de las instituciones humanas, p. ej., «Hay nieve en el Everest») y «hechos institucionales» (que sí requieren estados mentales, como p. ej., «Juan y yo estamos casados»).

Los hechos institucionales se definen mediante tres conceptos:

a) La intencionalidad colectiva, esto es, aquel tipo de estado mental intencional que se tiene sólo en tanto que compartido con otros individuos.

b) La asignación de funciones de status, es decir, la imposición de una función a algún objeto u hecho previamente existente, con la peculiaridad de que las características físicas de ese objeto no bastan por sí solas para cumplir esa función, sino que es necesaria la intencionalidad colectiva continuada al efecto. Se tiene un hecho institucional si y sólo si se asegura a un cierto tipo de objeto, por intencionalidad colectiva, una función que el objeto no está en condiciones de cumplir por su constitución física, sino sólo en virtud de la aceptación colectiva del hecho de que dicho objeto está en condiciones de cumplirla. SEARLE llama a este tipo de función «función de estatus», que puede expresarse como «X cuenta como $\mathrm{Y}$ en el contexto $\mathrm{C} »^{47}$.

45 A. RÓDENAS, 2006: «Sobre la validez jurídica: entre la normatividad y la convención», pp. 259-261.

${ }^{46}$ B. CELANO, 2000: «Fatti istituzionali e fatti convenzionali», pp. 163-164. Véase las obras de J. SEARLE, 1980: Actos de habla y, 1995: La construcción de la realidad social, y la de E. LAGERSPETZ, 1995: The Opposite Mirrors. An Essay on the Conventionalist Theory of Institutions.

${ }^{47}$ J. R. SEARLE, 1995: La construcción de la realidad social, pp. 23-46. 
c) Las reglas constitutivas, que son aquellas que no se limitan a regular un comportamiento ya existente, sino que constituyen su condición de posibilidad (como las reglas de un juego); las reglas constitutivas a la vez constituyen los hechos institucionales y describen su estructura lógica, según la fórmula «X cuenta como $\mathrm{Y}$ en el contexto $\mathrm{C} \gg^{48}$.

LAGERSPETZ considera su teoría como una mejora de la teoría de SEARLE de los hechos institucionales. La diferencia de fondo entre la teoría de SEARLE y la de LAGERSPETZ, radica en la noción de «convención» y en la de «conocimiento común». Según LAGERSPETZ la subsistencia de hechos dependientes de la creencia está sujeta a dos condiciones: 1) que se de un problema de interacción estratégica; 2) que la subsistencia del hecho en cuestión sea conciencia común entre los agentes implicados.

LAGERSPETZ se pregunta qué tipo de hecho es el hecho de que esas reglas existen en las comunidades relevantes. Obviamente no puede ser un hecho bruto (no es un hecho como la madera del mundo físico). Si la clasificación de SEARLE pretende ser una clasificación exhaustiva, los hechos relativos a las reglas deben ser por sí mismos hechos institucionales. Además, son inherentemente dependientes de la existencia de otras reglas: algo es una regla sólo si hay una regla con el efecto de que ese algo cuenta como una regla. Estamos en un regreso el infinito: el regreso lógico de las reglas ${ }^{49}$. De esta manera, la alternativa de LAGERSPETZ (frente a la critica que se hace a SEARLE del regreso lógico de las reglas) es construir una noción de hecho convencional que no esté fundada en reglas. Su idea básica es que hay cosas que existen y hechos que se sostienen sólo si los individuos relevantes creen que existen o los sostienen y actúan de acuerdo con estas creencias» $»^{50}$. Esto da lugar a la «creencia mutua compartida»

Tradicionalmente se asume que los hechos son independientes de nuestras creencias. Podemos tener razones para creer que ciertos hechos se dan, pero que los hechos realmente se den es independiente de estas razones. Por ejemplo, las montañas existen con independencia de nuestras razones para creer en su existencia. Esta distinción no funciona muy bien para la realidad social. Si todos nosotros creemos que estos pedazos de papel son dinero y que pueden ser intercambiados por bienes, son dinero que puede ser intercambiados por bienes. Esto tiene la consecuencia de que si produzco un argumento convincente (efectivo) es en el sentido de que si cierta regla es una regla jurídica válida, la regla es ipso facto una regla jurídica válida, porque el argumento ha producido la creencia relevante.

En definitiva, la noción de existencia convencional anular la distinción entre los hechos que son y nuestras creencias acerca de los mismos: en el terreno de los hechos sociales la creencia compartida en la existencia de una entidad convierte a tal entidad en existente: lo convencional constituye la realidad social ${ }^{51}$.

Es obvia la importancia de la idea de los hechos institucionales y el alcance de la misma en la teoría jurídica ${ }^{52}$; sin embrago, como señala NOGUERA, una cosa es decir que

48 J.A. NogUerA, 1999: «¿Son los hechos sociales una clase de hechos mentales?», pp.37-38.

49 E. LAGERSPETZ, 2001: «On the Existence of Institutions», p. 73.

${ }^{50}$ Cfr. A. RÓDENAS, 2006: «Sobre la validez jurídica», pp.259-260.

51 A. RÓDENAS, 2006: «Sobre la validez jurídica: entre la normatividad y la convención», p. 261.

${ }^{2}$ Cfr., por poner dos ejemplos las obras de E. LAGERPESTZ, 1995: The Opposite Mirrors. An Essay on the Conventionalist Theory of Institutions, y N. MACCORMICK y O. WEINBERGER, 1996: An Institutional Theory of Law. 
los hechos institucionales requieren de la existencia de alguna creencia humana para existir, y otra muy distinta decir que existen sólo porque creemos que existen ${ }^{53}$. A efectos de la existencia de las normas, creo que ésta debe definirse en los términos que he señalado antes: publicación en un diario oficial.

\section{BIBLIOGRAFÍA}

AARNiO, A., 1991: Lo racional como razonable, Madrid: Centro de Estudio Constitucionales.

Alchourrón, C. E., y Bulygin, E., 1979: Sobre la existencia de las normas jurídicas, Valencia (Venezuela): Facultad de Derecho-Univ. de Carabobo.

- 1991: «La concepción expresiva de las normas», en C. E. AlChOuRRón y E. BulYgin, Análisis lógico y derecho, Madrid: Centro de Estudios Constitucionales, pp. 121-153.

AlEXY, 1997: El concepto y la validez del derecho, Barcelona: Gedisa.

Betegón, J.; Gascón, M.; PÁramo, J.R., y DE Prieto, L., 1997: Lecciones de Teoría del Derecho, Madrid: MacGraw-Hill.

BовBIO, 1958: Teoria della norma giuridica, Torino: Giappichelli.

- 1960: Teoria dell 'ordinamento giuridico, Torino: Giappichelli.

Bulygin, E., 1991: «Validez y Positivismo», en C. E. AlChOurrón y E. Bulygin, Análisis lógico y derecho, Madrid: Centro de Estudios Constitucionales, pp. 499-519.

- 1991: «Tiempo y validez», en C. E. AlChOurRón y E. Bulygin, Análisis lógico y derecho, Madrid: Centro de Estudios Constitucionales, pp. 195-214.

— 1991: «Algunas consideraciones sobre los sistemas jurídicos», DOXA, n. ${ }^{\circ}$ 9, pp. 257-279.

CARACCIOLO, R., 1997: «Existencia de normas», Isonomia, n. ${ }^{\circ}$ 7, pp. 159-178.

- 1998: El sistema jurídico. Problemas actuales, Madrid: Centro de Estudios Constitucionales.

CELAnO, B., 2000: «Fatti istituzionali e fatti convenzionali», Filosofia e Questioni Pubbliche, 5 (2), pp. 163-164

CONTE, 1970: «Studio per una teoria della validità», Rivista Internazionale di Filosofia del Diritto, n. ${ }^{\circ} 47$, pp. 331-354.

ConTe, A.G., 1975: «Validità», Novisimo Digesto Italiano, vol. XX, pp. 418-425.

Delgado PiNTO, J., 1990: «Sobre la vigencia y validez de las normas», DOXA, n. ${ }^{\circ} 7$, pp. 101-167.

FERRAJOLI, L., 1995: Derecho y razón, Madrid: Trotta.

Ferrer Beltrán, J., 2000: Las normas de competencia, Madrid: Centro de Estudios Políticos y Constitucionales.

GASCÓN ABELLÁN, M., 1997: «Sentido y alcance de algunas distinciones sobre la invalidez de las leyes», DOXA, n. ${ }^{\circ} 20$, pp. 131-156.

GuAstinI, R., 1989: «Concetti e criteri di validità», Rivista Trimestrale di Diritto e Procedura Civile, 4, pp. 867-878.

- 1993: Le fonti del diritto e l'interpretazione, Milano: Giuffrè.

- 1994), «Invalidity», Ratio Iuris, vol.7, 2, pp.212-226.

- 1995: Il giudice e la legge, Padova: CEDAM.

- 1998: Teoria e dogmatica delle fonti, Milano: Giuffrè.

- 1999: Distinguiendo. Estudios de teoría y metateoría del derecho, Barcelona: Gedisa.

HERNÁNDEZ MARÍN, R., 2002: Introducción a la teoría de la norma jurídica, Madrid: Marcial Pons.

53 J.A. NoguerA, 1999: «¿Son los hechos sociales una clase de hechos mentales?», p. 49. 
JORI, M., 1993: «La cicala e la formica», en L. GIANFORMAGIO (coord.), Le ragioni del garantismo. Discutendo con Luigi Ferrajoli, Torino: Giappichelli, pp.66-119.

LagersPetz, E., 1995: The Opposite Mirrors, Dordrecht-Boston-London: Kluwer.

— 2001: «On the Existence of Institutions», en E. LAGERSPETZ, N. IKÄHEIMO y J. KOTKAVIRTA (eds.), On the Nature of Social and Institutional Reality, Sophi (Jyäskyla), pp.70-101.

LÓPEZ HERnÁNDEZ, J., 2004: «Existencia, validez y nulidad de las normas jurídicas», Anales de Derecho, n. ${ }^{\circ} 22$, pp. $99-120$.

MacCormick, N., 1978: Legal Reasoning and Legal Theory, Oxford: Clarendon Press.

MacCormick, N., y WeInBERGER, O., 1986: An Institutional Theory of Law, Dordrecth: Reidel.

MendoCA, D., 1992: Introducción al análisis normativo, Madrid: Centro de Estudios Constitucionales.

Moreso, J. J., 1996: «Lenguaje jurídico», en E. GARZÓN VALdÉs y F.J. LAPORTA (eds.), El derecho y la justicia, Madrid: Trota-CSIC-BOE, pp.106-107.

- 1997: Normas jurídicas y estructura del derecho, México: Fontamara.

- 1997: La indeterminación del derecho y la interpretación de la Constitución, Madrid: Centro de Estudios Políticos y Constitucionales.

Moreso, J.J., y Navaro, P., 1993: Orden jurídico y sistema jurídico, Madrid: Centro de Estudios Constitucionales.

Noguera, J.A., 1999: «¿Son los hechos sociales una clase de hechos mentales?», REIS, pp.35-60.

Ost, F., 1988: «Validité», en A.-J. ARNAud (dir.), Dictionnaire Enciclopedique de Theorie et de Sociologie du droit, Paris: LGDJ, pp. 431-435

Ost, F., y KeRCHOVE, M. VAN DE, 1987: Jalons pour una theorie critique du droit, Bruxelles: Facultes Universitaires Saint-Louis.

PeczeniK, A., y Hage, J., 1999: «Conocimiento jurídico, ¿’sobre qué?», DOXA, n. ${ }^{\circ} 22$, pp. 25-48.

PeÑA FreIRE, A. M., 1999: «Validez y vigencia de las normas: algunas precisiones conceptuales», Anuario de Filosofía del Derecho, XVI, pp.99-119

— 1999: «Reglas de competencia y existencia de las normas jurídicas», DOXA, n. ${ }^{\circ} 22$, pp. 381-412

— 2001: «Los sistemas jurídicos y la aplicabilidad de sus normas», Analisi e diritto, pp. 193-230

- 2003: «Las normas sobre la producción jurídica», Revista de Derecho de la Universidad de Granada, 6, pp. 119-154.

RAZ, J., 1982: La autoridad del derecho, México: UNAM.

REQUeJO PAGÉs, J. L., 1998: Las normas preconstitucionales y el mito del poder constituyente, Madrid: Centro de Estudios Políticos y Constitucionales.

RÓDENAS, A., 2006: «Sobre la validez jurídica: entre la validez y la convención», Analisi e diritto, pp. $255-276$

SEARLE, J.R., 1980: Actos de habla, Madrid: Cátedra.

- 1995: La construcción de la realidad social, Barcelona: Paidos.

Villajosana J. M., 1998: «Sobre recepción de normas», Isonomía, n. ${ }^{\circ}$ 8, pp. 181-191,

— 1998: «Dinámica de sistemas y persistencia de normas jurídicas», DOXA, n. ${ }^{\circ} 21-\mathrm{I}$, pp. 49-64.

WRIGHT, G.H. vON, 1970: Norma y acción, Madrid: Tecnos.

WRÓBLEWSKI, 1982: «Tre concetti di validità», Rivista Internazionale di Filosofia del Diritto, pp. 584-595.

- 1992: The Judicial Application of Law, Dordrecht-Boston-London: Kluwer. 
